\title{
Static skin effect in organic metals*
}

\author{
O.V. Kirichenko and V.G. Peschansky \\ B.I. Verkin Institute for Low Temperature Physics and Engineering \\ of the National Academy of Sciences of Ukraine, 47 Lenin Ave., Kharkov 61103, Ukraine \\ E-mail: vpeschansky@ilt.kharkov.ua
}

S.N. Savel'eva

A.F. Ioffe Physico-Technical Institute, Russian Academy of Sciences, St. Peterburg, Russia

Received December 19, 2002

\begin{abstract}
We have studied theoretically galvanomagnetic phenomena in layered conductors of organic origin with multi-sheet Fermi surface being of the form of a weakly corrugated cylinder and weakly corrugated planes. It is shown that in a strong magnetic field $\mathbf{H}$ unrestricted growth of the resistivity of such conductors with increasing $H$ is accompanied by forcing current lines out to the specimen surface. The main dissipation mechanism of electron flows is charge carriers scattering by sample boundary even in bulk conductors whose thickness is larger than conduction electrons free path length $l$. In the case of the specular reflection at the surface the resistivity grows linearly with the magnetic field increasing.
\end{abstract}

PACS: 72.15.Gd

Unrestricted growth of the resistivity of a conductor with increasing magnetic field is usually accompanied by forcing current lines out to the sample surface. In a strong magnetic field $\mathbf{H}$ when the curvature radius $r$ of electron trajectory is much less than the charge carriers free path $l$, electric current may be concentrated completely near the conductor surface (static skin effect) [1-6]. This takes place in compensated metals in which the numbers of electrons and «holes» are equal, and in metals with open Fermi surface at the magnetic field directions for which the resistivity increases with $H$. The reason is that charge carriers, colliding with the sample surface, show a higher mobility than volume electrons in the core of a conductor, because at every collision with the surface a jump of the center of electron orbit occurs. The surface current value depends essentially on the degree of the speculiarity of electron reflection at the sample boundary. This allows to use experimental studies of $H$-dependence of the conductor resistivity under the static skin-effect conditions as a method for checking the state of the surface without destruction of the specimen [7-12].
In metals the static skin effect is most pronounced in the case when the magnetic field vector is oriented parallel to the sample surface and orthogonal to the current density vector $(\mathbf{j} \perp \mathbf{H})$. This is the case when effective free path length $l_{\text {eff }}$ of electrons, that are reflected specularly by ideally smooth defectless sample surface, is limited by their collisions inside the volume only, i.e. $l_{\text {eff }}=l$, and the conductivity $\sigma_{\perp}^{\text {skin }}$ of the surface layer of the thickness $2 r$ is of the same order of magnitude as the conductivity $\sigma_{0}$ in the absence of a magnetic field. In compensated metals the contribution into the transverse conductivity $\sigma_{\perp}^{\mathrm{vol}}$ of interior electrons is of the order of $\sigma_{0}(r / l)^{2}$ and the electric field is orthogonal to the vector $\mathbf{H}$.

As a result, charge carriers that collide with the sample surface make the main contribution into the electric current density

$$
j_{i}=\sigma_{i k} E_{k}
$$

and the resistivity of the specimen with the thickness $d<<l^{2} / r$ grows linearly with $H$.

* The report was submitted at the 3rd International Workshop on Low Temperature Microgravity Physics (CWS-2002). 
In a magnetic field deflected from the sample surface at the angle $\alpha>l / r$, an electron goes into the bulk of a conductor after several collisions with the surface. Its effective free path $l_{\text {eff }}$ is much less than $l$ and equals $r / \sin \alpha$. The result is that the transverse resistivity $(\mathbf{j} \perp \mathbf{H})$ of compensated metals increases quadratically with a magnetic field at any relation between $d$ and $l$ even in the case of ideally smooth sample surface when specularly reflected electrons conserve their energy and projection of the momentum on the plane tangential to the surface.

Investigations of the surface state of layered conductors with the quasi-two-dimensional electron energy spectrum by means of measured magnetoresistance prove to be effective in magnetic fields inclined essentially from the specimen surface, as well.

We consider the distribution of current lines in organic conductors placed in a strong magnetic field in tetrathiafulvalene-based organic conductors $(\mathrm{BEDT}-\mathrm{TTF})_{2} \mathrm{X}$ with different radicals $\mathrm{X}$. This family of organic conductors represents layered structures with the sharp anisotropy of electrical conductivity of the metal type, the conductivity in the layers-plane being substantially greater than the conductivity across the layers. This apparently is connected with the sharp anisotropy of the conduction electrons velocity $\mathbf{v}=d \varepsilon(\mathbf{p}) / d \mathbf{p}$ on the Fermi surface $\varepsilon(\mathbf{p})=\varepsilon_{F}$, i.e. their energy

$$
\varepsilon(\mathbf{p})=\sum_{n=0}^{\infty} \varepsilon_{n}\left(p_{x}, p_{y}\right) \cos \left\{\frac{a n p_{z}}{\hbar}+\alpha_{n}\left(p_{x}, p_{y}\right)\right\},
$$

$$
\begin{aligned}
& \alpha_{n}\left(p_{x}, p_{y}\right)=-\alpha_{n}\left(-p_{x},-p_{y}\right), \\
& \varepsilon_{n}\left(p_{x}, p_{y}\right)=\varepsilon_{n}\left(-p_{x},-p_{y}\right)
\end{aligned}
$$

weakly depends on the momentum projection $p_{z}=\mathbf{p n}$ on a normal to the layers $\mathbf{n}$.

Here $a$ is the distance between the layers, $\hbar$ is the Planck constant, the functions $\varepsilon_{n}\left(p_{x}, p_{y}\right)$ decrease with $n$ increasing, such that the maximum value of the function $\varepsilon(\mathbf{p})-\varepsilon_{0}\left(p_{x}, p_{y}\right)$, being equal $\hbar \varepsilon_{F}$ at the Fermi surface, is much less than the Fermi energy $\varepsilon_{F}$.

The Fermi surface of layered conductors is open surface weakly corrugated along the $p_{z}$ axis. Experimental observation of the quantum Shubnikov-de Haas oscillations at first in complexes $(\mathrm{BEDT}-\mathrm{TTF})_{2} \mathrm{IBr}_{2}$ and $(\mathrm{BEDT}-\mathrm{TTF})_{2} \mathrm{I}_{3} \quad[13,14]$ and then in all tetrathiafulvalene-based layered conductors $[15,16]$ in a strong magnetic field $\mathbf{H}=(0, H \sin \theta, H \cos \theta)$ for a wide range of $\theta$ prove that at least one of the Fermi surface sheets represent a weakly corrugated cylinder.
In a magnetic field oriented parallel to the layers, a considerable part of electrons with the Fermi energy move along open orbits $\varepsilon=$ const, $p_{H}=\mathbf{p H} / H=$ const in the momentum space, and the resistance for the current flowing across the layers increases unlimitedly with $H$ increasing.

Let specimen be of the form of a plate with the thickness $d$ and boundaries $z_{s}=0, d$ and $y_{s}=0, L$, $d$ and $L$ being much greater than $l$. At $\theta=\pi / 2$ conduction electrons from the vicinity of the saddle point of the Fermi surface make the main contribution to the conductivity across the layers $\sigma_{z z}$ of the sample core [17], and the following formula is valid:

$$
\begin{gathered}
\sigma_{z z}^{\mathrm{vol}}=\sigma_{0} \gamma \eta^{2}, \eta^{1 / 2}<<\gamma<<1, \\
\sigma_{z z}^{\mathrm{vol}}=\sigma_{0} \gamma^{2} \eta^{3 / 2}, \quad \gamma \leq \eta^{1 / 2}<<1,
\end{gathered}
$$

where $\sigma_{0}$ is the conductivity in the absence of a magnetic field $[17,18]$. Its value is of the order of the conductivity of such metals as copper, gold, silver. Here and below $r=c p_{F} / e H$ is the curvature radius of an electron orbit at $\theta=0, \gamma=r / l, c$ is the velocity of light, $e$ is the electron charge, $p_{F}$ is the characteristic radius of the Fermi surface sheet which has the form of a weakly corrugated cylinder. If $L$ does not exceed much the free path length $l$, the contribution to $\sigma_{z z}$ from charge carriers «slipping» along the boundaries $y_{s}=0, L$ must be taken into account. These are conduction electrons with closed orbits. Their number related to the total number of charge carriers is not great (about $\eta^{3 / 2}$ ), but they possess higher mobility than electrons that do not contact the surface of the specimen. In the case of the specular reflection of electrons by the specimen boundary their drift along $z$ axis is limited by volume scattering only. The displacement of an electron along $z$ axis during the time between two collisions with the surface $y_{s}=0$ is about $r \eta^{1 / 2}$. For a small possibility $w$ of the diffuse scattering, at which the memory of the prior history of electrons is partly «erased», the effective free path of «slipping» electrons can be estimated as $l_{\text {eff }}=$ $=r \eta^{1 / 2} /(r / l+w)$ and conductivity of the boundary layer is of the form

$$
\sigma_{z z}^{\text {skin }}=\sigma_{0} \frac{l_{\text {eff }}}{l}=\sigma_{0} \frac{r \eta^{1 / 2} / l}{r / l+w} .
$$

The fractional number of electrons responsible for the formation of the skin layer is about $\eta^{3 / 2} r / L$ and the conductivity of the total specimen

$$
\sigma_{z z}=\sigma_{z z}^{\text {skin }} \eta^{3 / 2} r / l+\sigma_{z z}^{\mathrm{vol}}
$$

depends essentially on the state of the surface of a bulk specimen $(l<<L, d)$ in the range of very strong 
and, perhaps, unattainable now magnetic fields when $r / l<\eta^{2}$, only.

The resistance to the current flowing along the layers attains saturation in a strong magnetic field and is of the order of $1 / \sigma_{0}$. When the magnetic field is deviated from the layers-plane, all sections of the corrugated cylinder cut by the plane $p_{z}=$ const are closed and the resistance to the current flowing across the layers also saturates at $r<<l$.

Thus, in organic conductors whose Fermi surface represents a weakly corrugated cylinder and does not contain extra sheets, the distribution of the current lines is uniform over the total section normal to the current, and at any orientation of a magnetic field the conductivity is determined mainly by charge carriers that do not collide with the boundary.

The distribution of the current lines in a conductor whose Fermi surface consists of elements with different topological structure changes substantially. There are grounds to suppose that in organic complexes $(\mathrm{BEDT}-\mathrm{TTF})_{2} \mathrm{MHg}(\mathrm{SCN})_{4}$ (where $\mathrm{M}$ is either a metal of the group ( $\mathrm{K}, \mathrm{Rb}, \mathrm{Tl})$ or $\mathrm{NH}_{3}$ ) Fermi surface consists of a weakly corrugated cylinder and weakly corrugated planes [19]. In such conductors open sections of the Fermi surface cut by the plane $p_{z}=$ const occur at any orientation of a magnetic field and the resistivity saturates in a strong magnetic field for selected directions of the current only.

When the magnetic field makes an angle $\alpha=(\pi / 2-\theta)>>\eta$ with the layers-plane, electron trajectories in the momentum space are almost indistinguishable, and investigations of the interaction of charge carriers with the sample surface by measured magnetoresistance prove to be effective in a wide range of the angles $\alpha$.

Consider the case when the corrugated planes extend along the $p_{x} p_{y}$ plane, and the drift of electrons in the momentum space along the $p_{y}$ axis is limited. From the equations of motion

$$
\begin{gathered}
\frac{\partial p_{x}}{\partial t}=\frac{e H}{c}\left(v_{y} \cos \theta-v_{z} \sin \theta\right), \\
\frac{\partial p_{y}}{\partial t}=-\frac{e H v_{x}}{c} \cos \theta, \\
\frac{\partial p_{z}}{\partial t}=\frac{e H v_{x}}{c} \sin \theta
\end{gathered}
$$

for charge carriers that do not contact sample boundaries, it follows

$$
\overline{v_{x}}=\frac{1}{T} \int_{0}^{T} d t v_{x}(t)=0
$$

where $T=2 \pi m^{*} \mathrm{c} / \mathrm{eH}$ is the period of electron motion in a magnetic field, $m^{*}$ is its cyclotron effective mass. If motion along open orbit in the momentum space is not periodic, $T$ stands for a great enough time interval comparable with the free path time $\tau$.

Thus the contribution to the conductivity along the $x$ axis from volume electrons as with closed trajectories in the momentum space as with open ones, is inversely proportional to $H^{2}$, namely $\sigma_{x x}^{\mathrm{vol}} \cong \sigma_{0}(r / l)^{2}$.

The drift of electrons «slipping» near sample boundaries is different from zero in the $x$ axis direction also. Their contribution into the conductivity may appear to be principal. At $\theta$ different from $\pi / 2$ all orbits in the momentum space of electrons whose states belong to the corrugated cylinder sheet of the Fermi surface, are closed. Their drift along the $y$ axis is small. Colliding with the boundary $y_{s}=0$ the electrons drift for a long time along the $x$ axis and display slowly into the bulk of a conductor with the velocity $\overline{v_{y}}=\overline{v_{z}} \tan \theta$. At $\theta$ different essentially from $\pi / 2$, i.e. when $\tan \theta$ is of the order of unity, their effective free path has the form

$$
l_{\mathrm{eff}}=\frac{r}{r / l+w+\eta \tan \theta} .
$$

Since the quasi-two-dimensionality parameter for electron energy spectrum in organic layered conductors is of the order of $10^{-2}$, in a wide range for the angle $\theta(\eta \tan \theta<<1)$ the direct electric current is almost totally concentrated near the sample surface if

$$
L=\frac{l}{r / l+w+\eta \tan \theta} .
$$

Charge carriers that interact with the boundaries $z_{s}=0, d$ are also more mobile than electrons that «unaware» of the existence of the sample boundaries. The reflection of electrons from the boundaries $z_{s}=0, d$ is close to specular because they move slowly along the $z$ axis and approach these boundaries at small angles. Therefore a considerable part of the current is concentrated not only along the boundaries $y_{s}=0, L$ but also along the boundaries $z_{s}=0, d$. Conduction electrons approaching the boundaries $y_{s}=0, L$ at large angles, are at most capable of weakening the correlations between incident and reflected electrons. The possibility of the specular reflection of charge carriers at the boundaries $y_{s}=0, L$ can be easily determined by means of studying the resistivity of the layered conductor under the static skin effect conditions. 
1. M.Ja. Azbel, Zh. Eksp. Teor. Fiz. 44, 983 (1963).

2. M.Ja. Azbel and V.G. Peschansky, Zh. Eksp. Teor. Fiz. 49, 572 (1965).

3. M.Ja. Azbel and V.G. Peschansky, Zh. Eksp. Teor. Fiz. 52, 1003 (1967).

4. M.Ja. Azbel and V.G. Peschansky, Zh. Eksp. Teor. Fiz. 55, 1980 (1968).

5. G.I. Babkin and V.Ja. Kravchenko, Zh. Eksp. Teor. Fiz. 60, 695 (1971).

6. O.V. Kirichenko, V.G. Peschansky, and S.N. Savel'eva, Zh. Eksp. Teor. Fiz. 77, 2045 (1979).

7. O.A. Panchenko, A.A. Kharlamov, and Yu.G. Ptushinskii, Zh. Eksp. Teor. Fiz. 67, 780 (1974).

8. N.V. Volkenshtein, V.E. Startsev, and V.P. Dyakina, Phys. Status Solidi B66, K107 (1974).

9. Ju.P. Gaydukov, E.M. Galjamina, and N.P. Danilova, Pis'ma Zh. Eksp. Teor. Fiz. 22, 231 (1975).

10. Ju.P. Gaidukov and E.M. Galjamina, Zh. Eksp. Teor. Fiz. 75, 1426 (1978).

11. V.G. Peschansky, J. Stat. Phys. 38, 253 (1985).

12. V.G. Peschansky, Kinetic Size Effects in Metals in a Magnetic Field, Sov. Sci. Rev. 16, 1 (1992).
13. M.V. Kartsovnik, V.N. Laukhin, V.N. Nizhankovskii, and A.A. Ignat'ev, Pis'ma Zh. Eksp. Teor. Fiz. 47, 302 (1988)

14. M.V. Kartsovnik, P.A. Kononovich, V.N. Laukhin, and I.F. Shchegolev, Pis'ma Zh. Eksp. Teor. Fiz. 48, 498 (1988).

15. J. Wosnitza, Fermi Surfaces of Low-Dimensional Organic Metals and Superconductors, Springer Tracts in Modern Physics (1996), p. 165.

16. J. Singelton, Studies of Quasi-Two-Dimensional Organic Conductors Based on BEDT-TTF Using High Magnetic Fields, Report on Progress in Physics 116 (2000).

17. V.G. Peschansky, Zh. Eksp. Teor. Fiz. 112, 6183 (1997).

18. V.G. Peschansky, Phys. Rep. 288, 305 (1997).

19. R. Rossenau, M.L. Doublet, E. Canadell, R.P. Shibaeva, R.P. Rozenberg, N.D. Kushch, and E.B. Jagubskii, J. Phys. (France) 6, 1527 (1996). 